\title{
Frequency comb generation in silicon nitride resonators with amplitude modulated pump
}

\author{
J.M. Chavez Boggio*a, D. Bodenmüller ${ }^{\mathrm{a}}$, S. Ahmed ${ }^{\mathrm{a}}$, A. Baiga ${ }^{\mathrm{a}}$, T. Hansson ${ }^{\mathrm{b}}$, F. \\ Talentic, S. Wabnitz ${ }^{\mathrm{c}}$, and D. Modotto ${ }^{\mathrm{d}}$ \\ ainnoFSPEC - Leibniz Institut für Astrophysik Potsdam (AIP), An der Sternwarte 16, \\ D-14482, Potsdam, Germany; ${ }^{b}$ Department of Physics, Chemistry and Biology, \\ Linköping University, SE-581 83 Linköping, Sweden; 'Dipartimento di Ingegneria \\ dell'Informazione, Elettronica e Telecomunicazioni, Sapienza University of Rome, \\ 00184 Rome, Italy; ${ }^{\mathrm{D}}$ Dipartimento di Ingegneria dell'Informazione, Università di \\ Brescia, via Branze 38, 25123 Brescia, Italy
}

\begin{abstract}
We investigate frequency comb generation in silicon nitride ring resonators by using a pump subject to a weak amplitude modulation. We show that a partial locking is obtained when the external modulation frequency differs from the resonator free-spectral-range by up to hundreds of $\mathrm{MHz}$.
\end{abstract}

Keywords: Frequency comb, silicon nitride ring resonators, amplitude modulated pump, coherence.

\section{INTRODUCTION}

Optical frequency combs (OFCs) generated in micro resonators offer repetition rates spanning several orders of magnitude (from few GHz to THz) [1] [2] [3] [4] [5] [6] [7]. While those micro-resonators have been fabricated using a variety of materials and architectures, micro-ring resonators fabricated using the mature complementary metal oxide semiconductor (CMOS) technology are very promising, because of their potential for on-chip integration with other opto-electronic components. One CMOS material having a large cubic nonlinearity is silicon nitride $\left(\mathrm{SiX}_{\mathrm{X}} \mathrm{N}_{\mathrm{Y}}\right)[8,9]$, which has been largely explored for broadband and coherent frequency comb generation. Even though remarkably progress has been done over recent years, $\mathrm{SiX}_{\mathrm{X}} \mathrm{N}_{\mathrm{Y}}$ micro-resonators still face a number of issues that limit their use outside of laboratory settings. Fully coherent combs are obtained by accessing a soliton state through a complex pump wavelength tuning [10] [11] [12] [13]. In this standard procedure, a continuous wave $(\mathrm{CW})$ laser is used to pump a Six $\mathrm{N}_{\mathrm{Y}}$ resonator and generate a frequency comb through a cascade of four-wave mixing processes. However, this approach suffers from a very low use of the pump power (less than $1 \%$ of the pump power is converted into comb lines when single solitons are generated).

For this reason, the use of an amplitude-modulated pump has been recently theoretically [14] [15] [16] [17] [18] [19] [20] [21] as well as experimentally [22] [23] [24] [25] [26] [27] [28] studied. The aim here is to increase the conversion efficiency of comb generation, as well as to lock the frequency comb to the external modulation in order to stabilize the OFC. The mismatch between the external modulation frequency and the free spectral range (FSR) of the resonator has been investigated in order to elucidate the attainable locking range. Experiments demonstrated a soliton locking range of $\sim 60 \mathrm{kHz}$ when using a 1 ps pulse to pump a silica fiber Fabry-Perot resonator [25], for example. On the other hand, in [29] a fraction of the soliton light generated in a first resonator was injected into a second resonator, and a soliton with identical repetition rate than the one in the master resonator could still be generated, even when their FSRs were dissimilar by up to $\sim 8 \mathrm{MHz}$. In [30] a micro-resonator was pumped with two pumps $220 \mathrm{~nm}$ apart, to generate solitons that had their repetition rate synchronized thanks to cross-phase modulation (XPM), even when pumping different mode families with dissimilar dispersion values. It has also been reported that modulating a pump with a $20 \mathrm{MHz}$ signal resulted in the generation of soliton molecules with different constituent intensities, but with their repetition rates locked via XPM [31].

In this contribution, we investigate the generation of frequency combs when using asynchronous pump amplitude modulation in silicon nitride microring resonators. The modulation consist in weak sidebands that are $10 \mathrm{~dB}$ less intense than the main pump laser. We experimentally show that frequency 
comb states locked to both the external modulation and to the resonator FSR can be generated. This occurs when there is a mismatch between the modulation frequency and the resonator free-spectral range that is larger than the linewidth of the resonator resonance.

\section{EXPERIMENTAL SETUP}

Figure 1 shows the experimental setup. A CW tunable-laser with a short-term linewidth of $100 \mathrm{kHz}$ is used as a pump. Its light is amplitude modulated by using a $\mathrm{LiNbO}_{3}$ modulator driven by a radio frequency (RF) signal with a frequency nearly matching the FSR of our ring-resonator. With a bias voltage, the pump temporal shape can be adjusted by enhancing the intensity of the generated spectral sidebands. The inset of Figure 1 shows a typical modulated input spectrum where the first sidebands have $10 \mathrm{~dB}$ lower power than the pump laser line. The modulated light is then injected into an Erbium doped fiber amplifier (EDFA), where it can be amplified up to a nominal power of $4 \mathrm{~W}$. To filter out the amplified spontaneous emission noise from the EDFA, we use a band-pass optical filter having $0.4 \mathrm{~nm}$ bandwidth. The polarization state of the pump is adjusted with a polarization controller, in order to match the quasi-TE mode or the quasi-TM mode of the ring-resonator. The pump light is injected into the chip by using an objective lens (having NA 0.85). The generated frequency comb light is outcoupled from the chip using a lensed fiber. With a tunable Bragg optical filter, the pump is filtered out, and the filtered OFC is subsequently amplified with a low-noise pre-amplifier. Another optical filter having both tunable bandwidth and tunable center frequency is used to select a set of comb lines of the generated OFC, their beat-note detected with a photodiode (50 GHz bandwidth), and it is visualized with an electrical spectrum analyzer (ESA). This allows the measurement of the repetition rate coherence of the OFC. A Rubidium clock is used to discipline both the ESA and the signal generator.

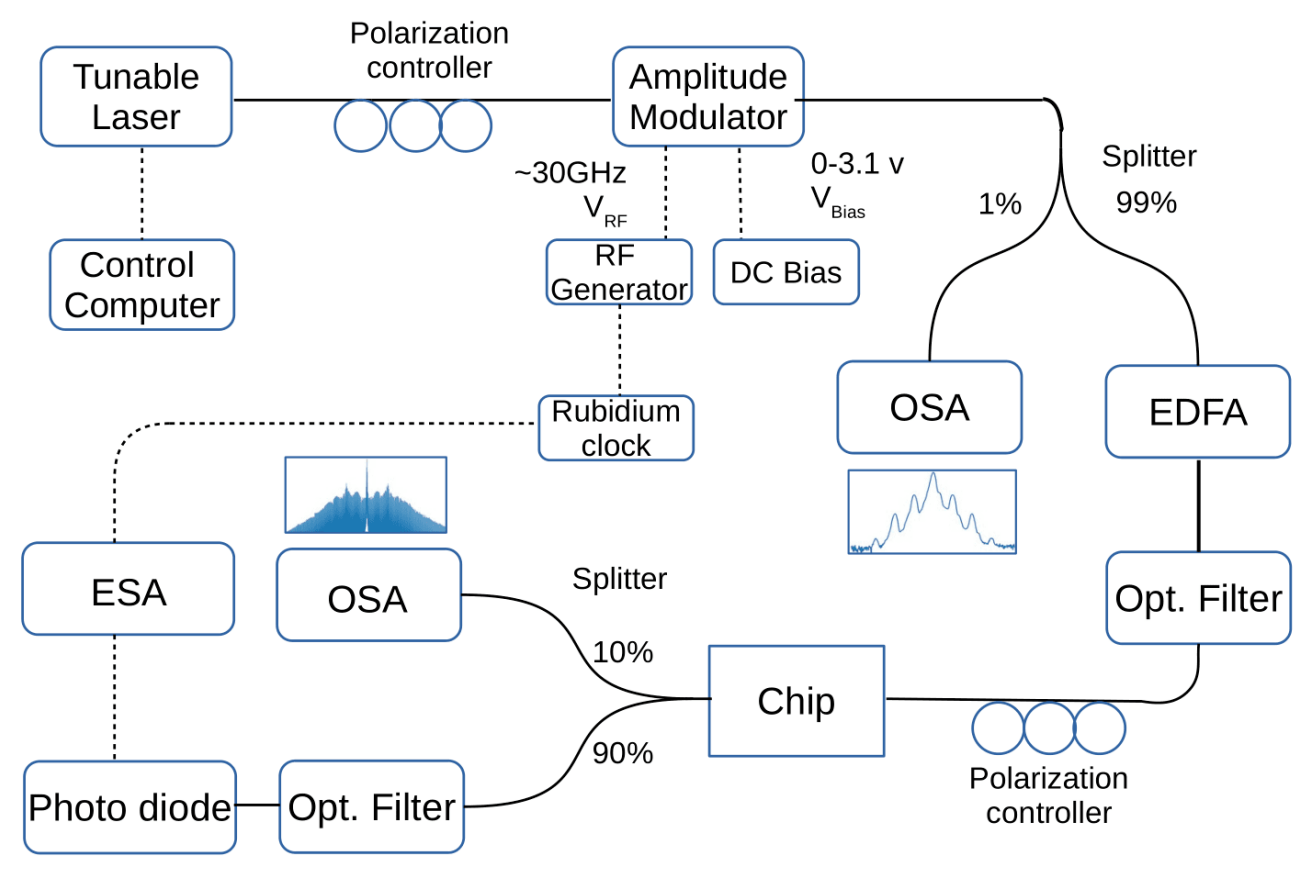

Figure1. Experimental setup for frequency comb generation with amplitude modulated pump.

A grating-based optical spectrum analyzer (OSA) having $20 \mathrm{pm}$ resolution was used to coarsely measure the frequency comb spectra. The experiments reported in this paper were done in two nearly identical ring resonators (A and B) having a measured $Q$-factor of $\sim 2 \times 10_{6}$ (resonance linewidth of $\sim$ $90 \mathrm{MHz}$ ), a radius of $800 \mu \mathrm{m}$, a core width $=1.7 \mu \mathrm{m}$ and a core height $=825 \mathrm{~nm}$, but differing in their gap values: gap $_{\mathrm{A}}=550 \mathrm{~nm}$, and gap $\mathrm{B}=600 \mathrm{~nm}$. The measured FSR were: $\mathrm{FSR}_{\mathrm{A}}=28.4925$ and FSR $\mathrm{B}=$ $28.4945 \mathrm{GHz}$. The resonators were fabricated in order to optimize frequency comb generation along the quasi-TE mode. 


\section{EXPERIMENTAL RESULTS}

Frequency comb generation when the mismatch between the external modulation and the FSR is smaller than $1.5 \mathrm{MHz}$ is investigated in Figure 2. The resonator B is pumped at an average power of $\sim 30 \mathrm{~mW}$, and the modulation frequency is increased, gradually approaching the FSR. There is a strong repetition rate peak at a frequency matching the modulation frequency, but sidebands are also present. The intensity and the separation of the sidebands decrease as the modulation frequency approaches the FSR of the resonator. When the mismatch between the external modulation frequency and the FSR is smaller than $\sim 200 \mathrm{kHz}$, sidebands collapse around the strong repetition rate peak and they are not noticeable anymore - this region is supposed to host coherent states. When the modulation frequency is larger than the FSR, a symmetrical behaviour occurs - the intensity and separation of the sidebands increase, and eventually the sidebands disappear when the mismatch between the modulation frequency and FSR exceeds $\sim 1.5 \mathrm{MHz}$. We further noticed that the intensity and position of the sidebands can be changed by varying the polarisation and the pump frequency detuning. These sidebands were also observed in resonator $\mathrm{A}$, and were also reported using $\mathrm{MgF}_{2}$ resonators [26]. This was attributed to a frequency pulling effect whereby all spectral components are pulled towards the FSR frequency. In the case of $\mathrm{MgF}_{2}$ resonators, the appearance of those sidebands occurs when the mismatch is in the $\mathrm{kHz}$ range (and the spacing between sidebands is of hundreds of $\mathrm{Hz}$ ): for a mismatch of hundreds of $\mathrm{Hz}$, those sidebands disappear, which corresponds to the soliton locking range.
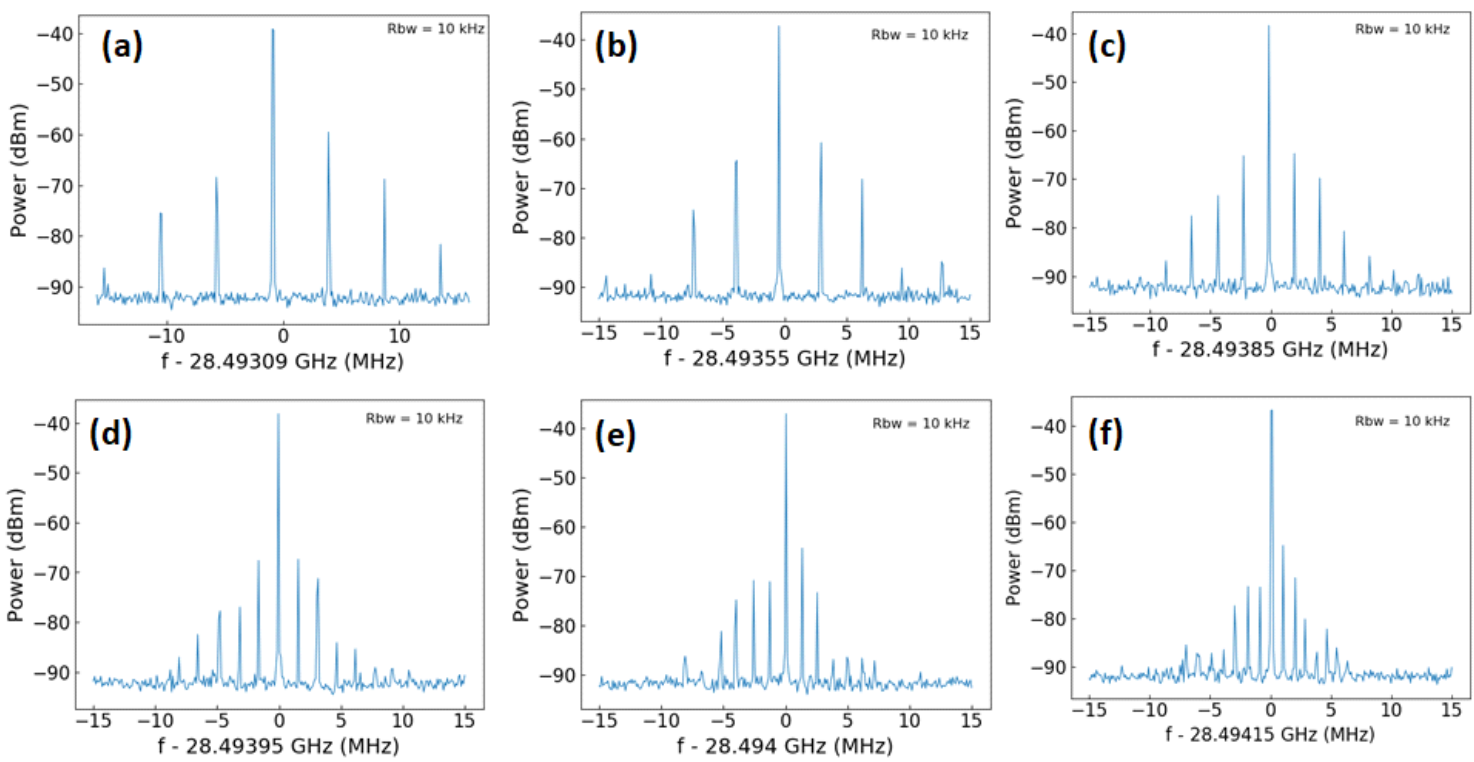

Figure 2. Frequency comb repetition rate for (a) 28.49309, (b) 28.49355, (c) 28.49385, (d) 28.49395, (e) 28.494 and (f) $28.49415 \mathrm{GHz}$. The FSR of the resonator is $28.4945 \mathrm{GHz}$.

We experimentally analyzed the repetition rate of the frequency comb for larger mismatches between the external modulation frequency and the FSR of the resonator. This was done for both relatively higher $(\sim 400 \mathrm{~mW})$ and lower $(\sim 50 \mathrm{~mW})$ pump power values. Figure 3 shows the results of these measurements. For the $400 \mathrm{~mW}$ case we generated mostly chaotic MI comb states, and the detected repetition rate signal exhibits a narrow peak sitting on a noise bump. The repetition rate peak has a frequency that is given by the external amplitude modulation. The narrow peak is still visible even for modulation frequencies almost $1 \mathrm{GHz}$ away from the FSR, indicating that a (small) fraction of the frequency comb power is (partially) locked to the amplitude modulation. For the $50 \mathrm{~mW}$ case the range of partial locking is smaller.

The large partial locking that is seen in the results of Figure 3 might be explained either by thermal or nonlinear effects. The heating in the $\mathrm{SiX}_{\mathrm{X}} \mathrm{N}_{\mathrm{Y}}$ resonator produces a shift of the resonance frequency of approximately 3-5 GHz, which corresponds to a change of the FSR of the resonator of $\sim 8 \mathrm{MHz}$ [2] (two orders of magnitude smaller than the partial locking range we measured in Figure 3). A different source of resonance mode shift comes from the nonlinear refractive index dependence on intra-cavity power (via Kerr nonlinearity). 

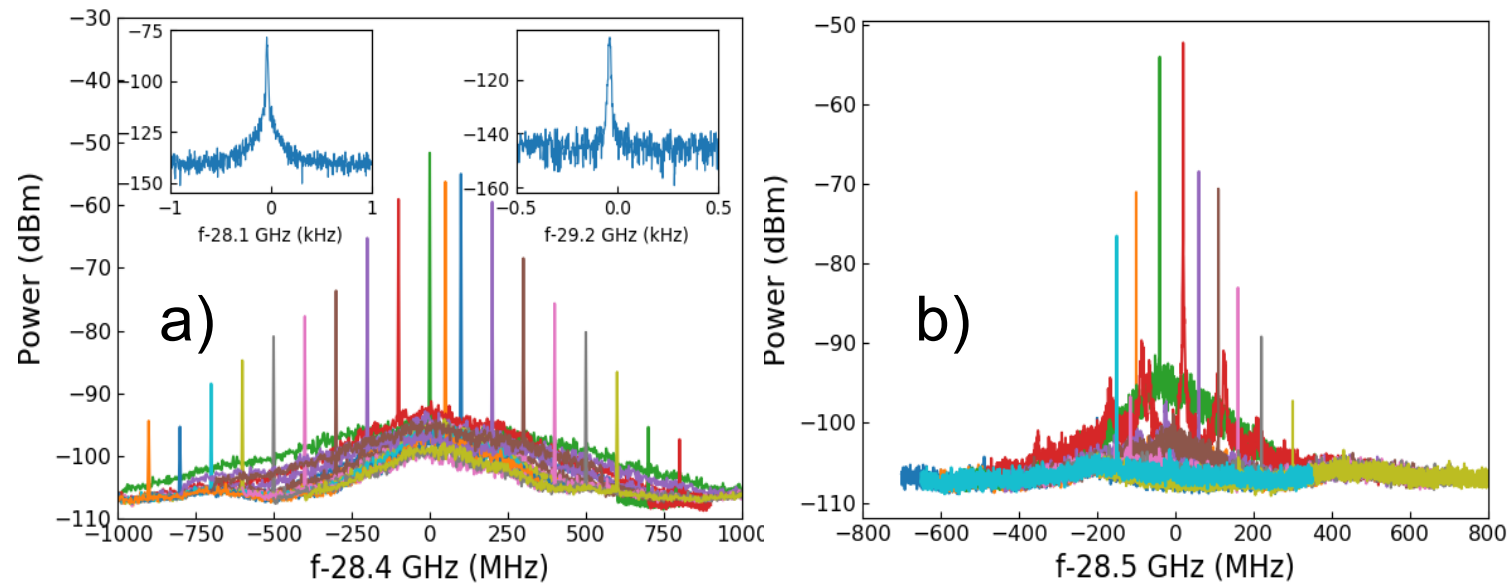

Figure 3. Repetition rate of the frequency comb as the modulation frequency is tuned (a) $P \sim 400 \mathrm{~mW}$. Inset shows the zoomed repetition rate obtained with a resolution bandwidth of $1 \mathrm{~Hz}$ (b) $P \sim 50 \mathrm{~mW}$.

Figure 4 shows the calculation of the Kerr tilted resonance for a $\mathrm{SiXN}_{\mathrm{X}}$ resonator having the same parameters as in our experiment in Fig. 3(a) $\left(\mathrm{P}=400 \mathrm{~mW}\right.$ in the bus waveguide, $\mathrm{Q}=2 \times 10^{6}$, etc). Note that the resonance is pulled by almost $1 \mathrm{GHz}$, which matches the locking range that we have experimentally observed.

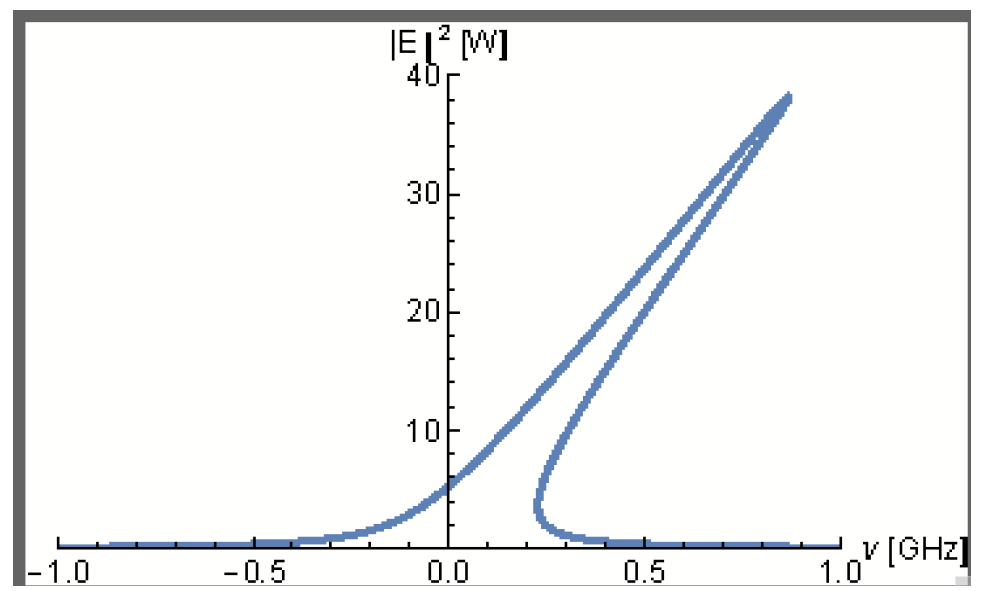

Figure 4. Calculated Kerr tilted resonance for the $\mathrm{Si}_{\mathrm{X}} \mathrm{N}_{\mathrm{Y}}$ resonator with $P=400 \mathrm{~mW}$ and $Q=2 \times 10^{6}$.

The fact that the intensity of the repetition signal decreases when the mismatch between the modulation frequency and the FSR increases can be attributed to a filtering effect. As the mismatch increases the modulation component is mostly filtered out, and pumping is $\mathrm{CW}$ to a great extent. Furthermore, at higher pump powers mode pulling due to the Kerr effect is also larger, resulting in an extended partial locking range.

Some frequency comb spectra exhibit rather ordered/periodic shape, which might be associated to partially coherent states. When measuring their repetition rate signal, a mix of peaks associated to the FSR and the external modulation frequencies appears. The more ordered/periodic the comb spectrum is, the narrower/sharper are the repetition rate peaks. Figure 5(a) shows the frequency comb spectrum generated by pumping resonator A with $400 \mathrm{~mW}$ power at a modulation frequency of $29.1 \mathrm{GHz}$, where the combined effects of the FSR and the external modulation are likely to be present. For this modulation, the mismatch with the FSR is of $606 \mathrm{MHz}$, which is much larger than the resonance linewidth. Figure 5(b) shows the repetition rate spectrum, where a strong signal at $28.494 \mathrm{GHz}$ can be readily observed, together with a weaker peak at $29.1 \mathrm{GHz}$. 

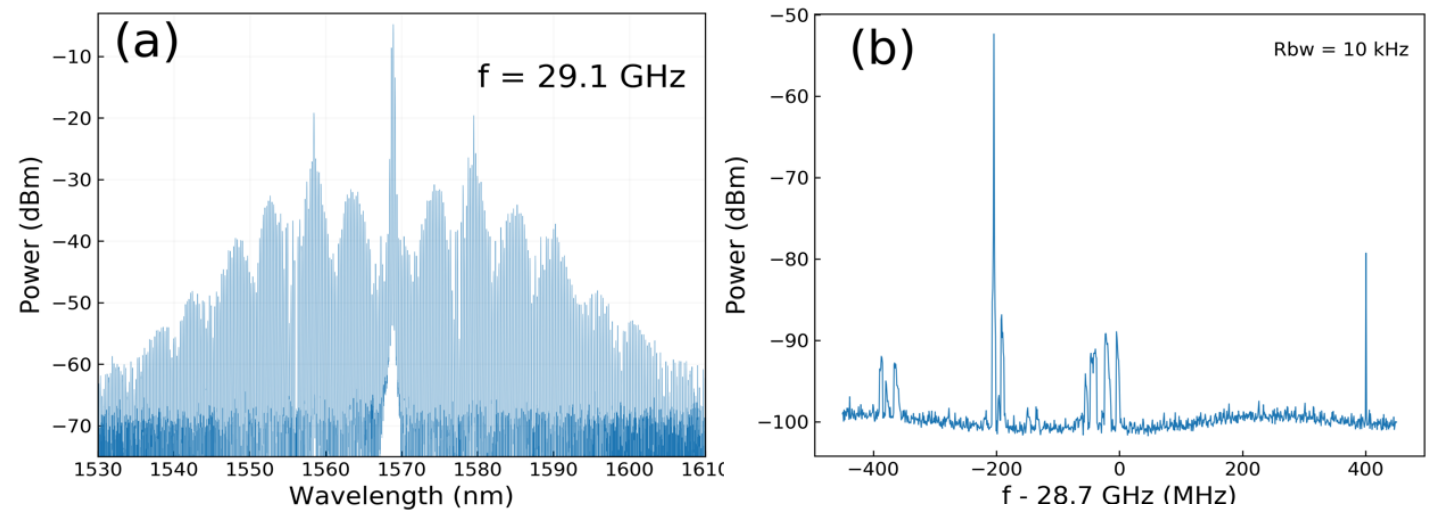

Figure 5. (a) Frequency comb spectrum and corresponding (b) repetition rate signal showing locking to both the FSR (peak at $\sim-200 \mathrm{MHz}$ ) and to the external modulation (peak at $\sim 400 \mathrm{MHz}$ ).

Figure 6(a) shows a rather incoherent case, where the amplitude modulation was set to $f=28.8 \mathrm{GHz}$. We highlight with a black arrow the repetition rate corresponding to the external modulation frequency. Whereas the red arrow highlights the repetition rate associated with the FSR of the ring resonator. Additional, less intense spectral components are also generated. With dashed lines, we indicate spectral components that are symmetrically generated around the main peak corresponding to the FSR of the resonator. Figure 6(b) shows the corresponding frequency comb spectrum.
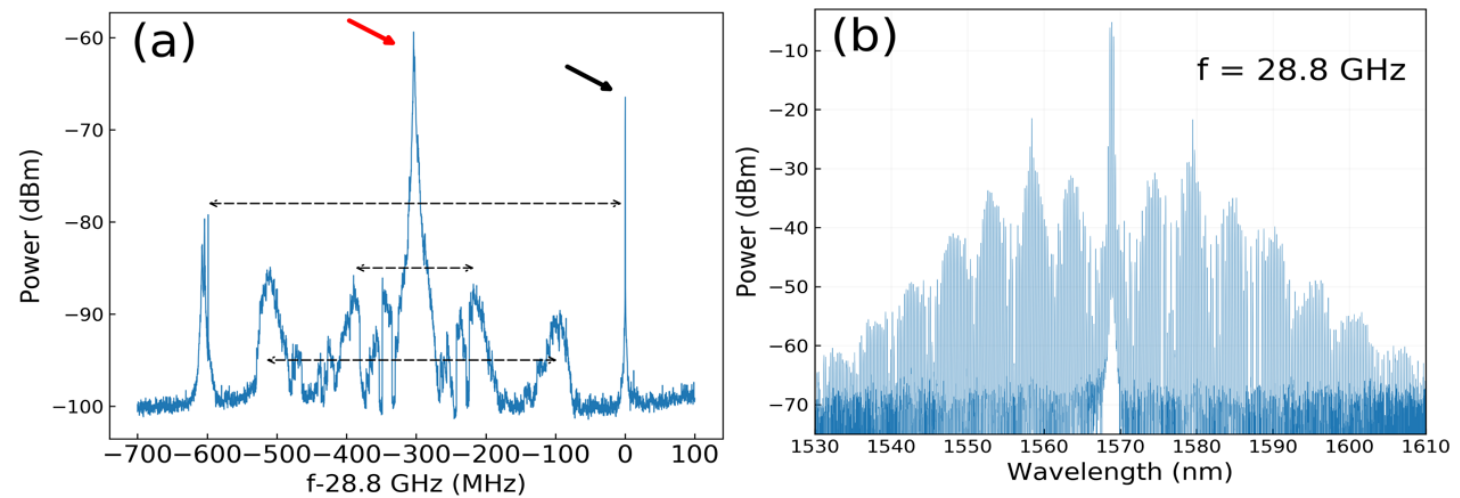

Figure 6. (a) Repetition rate signal and corresponding (b) frequency comb spectrum.

A qualitative explanation of the observed dynamics is as follows. As the pump is tuned into a resonance, the primary MI sidebands are generated several FSRs outside the pump frequency. There is then a secondary sub-combing formation around the primary lines having the repetition rate of the external amplitude modulation. This is finally followed by a merging and synchronization of the subridges. This is a Type 2 comb where the amplitude modulation is seeding the sub-comb formation rather than the primary sidebands. The persistent observation in the repetition rate signal of a component coming from the external modulation, even when the frequency of the modulation differs from the FSR by hundreds of $\mathrm{MHz}$, arises from the seeded sub-comb formation.

\section{CONCLUSIONS}

Frequency comb generation in silicon nitride ring-resonators was investigated when using asynchronous pump amplitude modulation. We experimentally showed that frequency comb states partially locked to the external modulation can be generated when there is a mismatch between the modulation frequency and the resonator FSR larger than the linewidth of the resonator resonance. The partial locking range for the generation of these states is shown to depend on the pump power, likely due to a resonance tilt coming from Kerr nonlinearity.

For modulation frequencies very close to the FSR (with a mismatch smaller than $1.5 \mathrm{MHz}$ ), a 
frequency comb regime is described showing the generation of multiple sidebands around the main repetition rate, which collapse towards the main repetition rate peak as the modulation frequency approaches the FSR. Finally, we described a regime of comb generation that exhibits two repetition rates, one linked to the external modulation, and the other linked to the FSR of the resonator, the latter one being very noisy.

\section{Acknowledgments}

This work is supported by BMBF (Federal Ministry of Education and Research) through grants 03Z2AN11 and 03Z2AN12. T.H. acknowledges funding from the Swedish Research Council (Vetenskapsrådet, Grant. No. 2017-05309). F.T. and S.W. acknowledge funding from the European Union's Horizon 2020 Research and Innovation Programme under the Marie Skłodowska-Curie ITNEID MOCCA (Grant No. 814147).

\section{REFERENCES}

[1] Kippenberg, T. J., Holzwarth, R. and Diddams, S. A., "Microresonator based optical frequency combs, " Science 332, 555-559 (2011).

[2] Okawachi, Y., Saha, K., Levy, J. S., Wen, Y. H., Lipson, M. and Gaeta, A. L., "Octave-spanning frequency comb generation in a silicon nitride chip," Opt. Lett., 36, 3398-3400 (2011).

[3] Ferdous, F., Miao, H., Leaird, D. E., Srinivasan, K., Wang, J., Chen, L., Varghese, L. T. and Weiner, A. M., "Spectral line-by-line pulse shaping of on-chip microresonator frequency combs," Nature Photon. 5, 770-776 (2011).

[4] Herr, T., Hartinger, K., Riemensberger, J., Wang, C.Y., Gavartin, E., Holzwarth, R., Gorodetsky, M. L. and Kippenberg, T. J., "Universal formation dynamics and noise of Kerr-frequency combs in microresonators," Nature Photon. 6, 480-487 (2012).

[5] Papp, S. B., Beha, K., Del'Haye, P., Quinlan, F., Lee, H., Vahala, K. J., and Diddams, S. A., "Microresonator frequency comb optical clock," Optica 1, 10-14 (2014).

[6] Erkintalo, M. and Coen, S., "Coherence properties of Kerr frequency combs.," Opt. Lett. 39, 283-286 (2014).

[7] Yi. X, Yang. Q.-F, Yang. K. Y, Suh. M.-G, and Vahala. K., "Soliton frequency comb at microwave rates in a high-Q silica microresonator," Optica 2, 1078-1085 (2015).

[8] Chávez Boggio, J. M., Ortega Moñux, A., Modotto, D., Fremberg, T., Bodenmüller, D., Giannone, D., Roth, M. M., Hansson, T., Wabnitz, S., Silvestre, E. and Zimmermann, L., "Dispersion-optimized multicladding silicon nitride waveguides for nonlinear frequency generation from ultraviolet to midinfrared," J. Opt. Soc. Am. B 33, 2402-2413 (2016).

[9] Chavez Boggio, J. M., Bodenmüller, D., Fremberg, T., Haynes, R., Roth, M. M., Eisermann, R., Lisker, M., Zimmermann, L. and Böhm, M., "Dispersion engineered silicon nitride waveguides by geometrical and refractive-index optimization," J. Opt. Soc. Am. B 31, 2846-2857 (2014).

[10] Herr, T., Brasch, V., Jost, J. D., Wang, C. Y., Kondratiev, N. M., Gorodetsky, M. L., and Kippenberg, T. J., "Temporal solitons in optical microresonators," Nat. Photonics 8, 145-152 (2014).

[11] Guo, H., Karpov, M., Lucas, E., Kordts, A., Pfeiffer, M. H. P., Brasch, V., Lihachev, G., Lobanov, V. E., Gorodetsky, M. L. and Kippenberg, T. J., "Universal dynamics and deterministic switching of dissipative Kerr solitons in optical microresonators," Nat. Phys. 13, 94-102 (2017).

[12] Jaramillo-Villegas, J. A., Xue, X. X., Wang, P. H., Leaird, D. E. and Weiner, A. M., "Deterministic single soliton generation and compression in microring resonators avoiding the chaotic region.," Opt. Express 23, 9618-9626 (2015).

[13] Liu, J., Lucas, E., Raja, A. S., He, J., Riemensberger, J., Wang, R. N., Karpov, M., Guo, H., Bouchand, R. and Kippenberg, T. J., "Photonic microwave generation in the X- and K-band using integrated soliton microcombs," Nat. Photonics 14, 486-491 (2020).

[14] Hansson, T. and Wabnitz, S., "Bichromatically pumped microresonator frequency combs," Phys. Rev. A 90, 13811 (2014).

[15] Parra-Rivas, P., Gomila, D., Matias, M. A., Colet, P., and Gelens, L., "Effects of inhomogeneities and drift on the dynamics of temporal solitons in fiber cavities and microresonators," Optics Express A 22, 30943 (2014).

[16] Lobanov, V. E., Lihachev, G. V., Pavlov, N. G., Cherenkov, A. V., Kippenberg, T. J. and Gorodetsky, M. L., "Harmonization of chaos into a soliton in Kerr frequency combs," Opt. Express 24(24), 27382-27394 (2016). 
[17] Taheri, H., Matsko, A. B. and Maleki, L., "Optical lattice trap for Kerr solitons," Eur. Phys. J. D 71, 153 (2017).

[18] Hendry, I., Chen, W., Wang, Y., Garbin, B., Javaloyes, J., Oppo, G.-L., Coen, S., Murdoch, S. G. and Erkintalo, M., "Spontaneous symmetry breaking and trapping of temporal Kerr cavity solitons by pulsed or amplitude-modulated driving fields," Phys. Rev. A 97(5), 053834 (2018).

[19] Hendry, I., Garbin, B., Murdoch, S. G., Coen, S. and Erkintalo, M., "Impact of desynchronization and drift on soliton-based kerr frequency combs in the presence of pulsed driving fields," Physical Review A 100,023829 (2019).

[20] Weiblen, R. J. and Vurgaftman, I., "Bichromatic pumping in mid-infrared microresonator frequency combs with higher-order dispersion," Opt. Express 27, 4238-4260 (2019).

[21] Lobanov, V. E., Kondratiev, K. M., Shitikov, A. E., Galiev, R. R. and Bilenko, I. A., "Generation and dynamics of solitonic pulses due to pump amplitude modulation at normal group-velocity dispersion," Phys. Rev. A 100, 013807 (2019).

[22] Strekalov, D.V. and Yu, N., "Generation of optical combs in a whispering gallery mode resonator from a bichromatic pump," Phys. Rev. A, 79(4):041805, 2009.

[23] Papp, S. B., Del'Haye, P. and Diddams, S. A., "Parametric seeding of a microresonator optical frequency comb," Opt. Express 21(15), 17615-17624 (2013).

[24] Lin, G., Martinenghi, R., Diallo, S., Saleh, K., Coillet, A. and Chembo, Y. K., "Spectro-temporal dynamics of Kerr combs with parametric seeding,"Applied Optics Vol. 54, Issue 9, pp. 2407-2412 (2015).

[25] Obrzud, E., Lecomte, S. and Herr, T., "Temporal solitons in microresonators driven by optical pulses," Nat. Photonics 11, 600 (2017).

[26] Weng, W., Lucas, E., Lihachev, G., Lobanov, V. E., Guo, H., Gorodetsky, M. L. and Kippenberg, T. J., "Spectral Purification of Microwave Signals with Disciplined Dissipative Kerr Solitons," Phys. Rev. Lett. 122, 013902, 2019.

[27] Anderson, M., Bouchand, R., Liu, J., Weng, W., Obrzud, E., Herr, T. and Kippenberg, T. J., "Photonic chip-based resonant supercontinuum generation with intrinsic nonlinear filtering," in Conference on Lasers and Electro-Optics, OSA Technical Digest (Optical Society of America, 2020).

[28] Yiqing Xu, Yi Lin, Alexander Nielsen, Ian Hendry, Stéphane Coen, Miro Erkintalo, Huilian Ma, and Stuart G. Murdoch, "Harmonic and rational harmonic driving of microresonator soliton frequency combs," Optica 7, 940-946 (2020).

[29] Jang, J. K., Klenner, A., Ji, X., Okawachi, Y., Lipson, M. and Gaeta, A.L., "Synchronization of coupled optical microresonators," Nature Photon 12, 688-693 (2018).

[30] Zhang, S., Silver, J.M., Bi, T. et al., "Spectral extension and synchronization of microcombs in a single microresonator," Nat Commun 11, 6384 (2020).

[31] Weng, W., Bouchand, R., Lucas, E., Obrzud, E., Herr, T. and Kippenberg, T.J., "Heteronuclear soliton molecules in optical microresonators," Nat. Commun. 11, 2402 (2020).

[32] Karpov, M., Pfeiffer, M. H. P., Guo, H., Weng, W., Liu, J. and Kippenberg, T. J., "Dynamics of soliton crystals in optical microresonators," Nat. Phys. 15, 1071-1077 (2019).

[33] Wang, W., Lu, Z., Zhang, W., Chu, S.T., Little, B. E., Wang, L., Xie, X., Liu, M., Yang, Q., Wang, L., Zhao, J., Wang, G., Sun, Q., Liu, Y., Wang, Y. and Zhao, W., "Robust soliton crystals in a thermally controlled microresonator," Opt. Letters 43, 2002-2005, (2018).

[34] Lucas, E., Brochard, P., Bouchand, R., Schilt, S., Südmeyer, T. and Kippenberg, T. J., "Ultralownoise photonic microwave synthesis using a soliton microcomb-based transfer oscillator," Nat. Commun. 11, 374 (2020).

[35] Razavi, B., "A study of injection locking and pulling in oscillators," IEEE Journal of Solid-State Circuits 39, 1415-1424 (2004). 